\title{
La práctica pedagógica en la fase antes de la lectura en clases de comprensión de textos en enseñanza media*
}

\author{
Carolina Iturra Herrera ${ }^{* *}$ \\ Elizabeth Donoso Osorio*** \\ Ivonne Fuentes Román***
}

\begin{abstract}
Resumen
Este trabajo analizó la fase antes de la lectura en clases de enseñanza media para identificar prácticas docentes vinculadas con la enseñanza de la comprensión de textos y promoción de estrategias metacognitivas. Se seleccionaron 30 clases del sistema de evaluación del desempeño docente 2009 y 2013, las que fueron estudiadas utilizando un sistema de análisis del discurso considerando el tipo de tarea incluida, el patrón comunicativo y las elaboraciones públicas construidas. Los resultados arrojaron patrones afines con la secuencialidad en las tareas, tipos de patrones comunicativos y elaboraciones públicas en cada tarea que contribuirían al modelaje de las estrategias cognitivas, detectándose solo diferencias en los contenidos de las tareas de planificación, en tanto los patrones restantes se mantuvieron similares.
\end{abstract}

Palabras clave: Análisis discurso educativo, enseñanza de la comprensión de textos, estrategias metacognitivas de comprensión de textos.

\section{The pedagogical practice in the phase before reading in text comprehension classes in high school}

\begin{abstract}
This work analyzed the before reading phase in middle school classes to identify teaching practices linked to the teaching of text comprehension and promotion of metacognitive strategies. Thirty classes of the 2009 and 2013 teaching performance evaluation system were selected. These were studied using a system of discourse analysis that considered the type
\end{abstract}

Proyecto de investigación Fondecyt 1160205 “Caracterización de las prácticas instruccionales de la enseñanza de la comprensión de textos, en segundo ciclo de enseñanza general básica y enseñanza media, a partir de la evidencia audiovisual de la evaluación docente". Se agradece al Centro de Perfeccionamiento, Experimentación e Investigaciones Pedagógicas (CPEIP) y al Centro de Investigación en Ciencias Cognitivas de la Universidad de Talca.

"* Chilena. Doctora en Psicología Educacional. Profesora de Universidad de Talca, Talca, Chile. citurra@utalca.cl

*** Chilena. Doctora en Instrucción y Currículum. Académica de la Pontificia Universidad Católica de Valparaíso, Valparaíso, Chile. elizabeth.donoso@pucv.cl

*** Chilena. Doctora en Lingüística. Profesora asociada de la Universidad de Playa Ancha, Valparaíso, Chile.ifuentes@upla.cl 
of task included, the communicative pattern and the public production constructed. The results showed patterns linked to the sequentiality of the tasks, types of communicative patterns and public production in each task, which could contribute to the modeling of cognitive strategies, detecting only differences in the contents of the planning tasks, while the rest of the patterns remained similar.

Keywords: Educational discourse analysis, Metacognitive strategies for text comprehension, Teaching text comprehension.

\section{Introducción}

Uno de los grandes desafíos que enfrentan los países de Latinoamérica es lograr la alfabetización de los estudiantes en los distintos niveles de enseñanza. En este sentido, la United Nations Educational, Scientific and Cultural Organization, Unesco (2015) plantea que esta alfabetización implica educar a las nuevas generaciones, otorgándoles herramientas que les permitan incorporarse al mundo del siglo XXI, mediante el aprendizaje de competencias más complejas. Una de estas es la competencia lectora que puede definirse como "la capacidad de comprender, utilizar y analizar textos escritos para alcanzar los objetivos del lector, desarrollar sus conocimientos y posibilidades y participar en la sociedad" (Organización para la Cooperación y el Desarrollo Económicos, OCDE, 2006, p. 13).

Por tanto, este proceso involucraría numerosas habilidades y estrategias que permitirían a un lector construir significados y conocimientos a partir de las ideas de un texto (Anderson \& Pearson, 1984; Kintsch, 1998; Myers \& O’Brien, 1998).

En este escenario, hoy se plantea la necesidad de apoyar a los estudiantes para que alcancen aprendizajes más allá de la decodificación lectora o la comprensión literal, dado que la lectura se define como una competencia que se desarrolla y complejiza a lo largo de la vida, asumiendo entonces que este aprendizaje es "amplio, multidimensional, que requiere la movilización de capacidades cognitivas, afectivas y de inserción social" (Solé, 2012, p. 59). En este sentido, se asume que la capacidad para comprender aquello que se lee descansa sobre ciertos procesos básicos y de alto nivel, los que permiten a los lectores competentes acceder a la información de los variados textos que leen. Al respecto, existen evidencias empíricas que remarcan que uno de los focos 
principales en esta alfabetización debiera ser la enseñanza de estrategias y de su uso, con el propósito de lograr lectores estratégicos (Paris, Wasik \& Tuner, 1991), beneficiando así la capacidad de los estudiantes para elaborar, organizar y evaluar aquello que leen y fomentando su pensamiento y aprendizaje continuo. Desde esta perspectiva, cobra importancia la enseñanza explícita de estas estrategias a partir de experiencias de lectura conjunta (Sánchez, García y Rosales, 2010), que serían situaciones de aprendizaje diseñadas por los profesores para desplegar acciones intencionadas que apoyen el aprendizaje de la comprensión de textos, no obstante, aun cuando se reconoce un avance considerable en la investigación acerca del fenómeno de la comprensión de textos, el cuerpo de conocimientos respecto de cómo se enseña esta competencia en las aulas es menor, así como cuáles son las estrategias y prácticas que los profesores promueven para potenciar el aprendizaje y uso de estrategias cognitivas. Considerando esto, se requiere contar con trabajos que permitan describir cómo se desarrollan estos procesos en las aulas.

\section{Referentes teóricos}

La comprensión de textos es un fenómeno complejo que requiere del uso de distintos procesos y estrategias que beneficiarían la capacidad para entender, organizar y construir las representaciones que elaboramos a partir del material leído. En este sentido, se reconoce la importancia de aprender a desplegar estrategias y metaestrategias cognitivas que facilitarían, entre otras cosas, su uso de manera flexible y selectiva como herramientas que permitan extraer ideas de los textos, fomentando la lectura y el aprendizaje, además de promocionar la capacidad estratégica, beneficiando el entendimiento de diversas áreas del conocimiento (Paris et al., 1991). De esta manera, los procesos que activamos frente a la tarea de comprender un texto implicarían distintas acciones que deben integrarse y autorregularse, permitiendo así un mayor control sobre los procesos implicados en ella (Madariaga y Martínez-Villabeita, 2010).

Para lograr esta autorregulación, los lectores expertos utilizan estrategias metacognitivas, entendidas estas como la capacidad que poseen los sujetos para ser conscientes de su propio conocimiento y la forma en que operan cognitivamente los procesos que les permiten acceder y construir dichos saberes (Flavell, 1976). Considerando el 
dominio específico de la comprensión de textos, esto implicaría desplegar acciones dirigidas a regular la activación de conocimientos previos necesarios para hacer frente a la tarea (Brown, 1987) y utilizar estrategias para planificar, monitorear y evaluar el logro de la comprensión (Baker \& Carter, 2009). Ello requiere que los aprendices manejen estrategias de aprendizaje que les permitan una adecuada toma de decisiones para seleccionar y usar procedimientos que faciliten el proceso de lectura, de manera intencional y autorregulada (Trabasso \& Bouchard, 2002).

$\mathrm{Al}$ respecto, se señala la importancia de la enseñanza y modelaje de estrategias metacognitivas que fomentarían la capacidad para establecer metas y enfrentar tareas de lectura cada vez más complejas. Frente a esto, hoy se cuenta con evidencia empírica que describe cómo se logra un mayor nivel de comprensión de lectura (Slavin, Lake, Chambers, Cheung \& Davis, 2009) cuando se instruye a los estudiantes en la aplicación de estrategias de metacognitivas (por ejemplo, la generación de preguntas o resumir).

Así, autores como Schmitt y Bauman (1990), Solé (2008) y Block y Presley (2007) entre otros, han identificado distintas estrategias metacognitivas que deben ser usadas en el momento antes de la lectura, como por ejemplo, identificar el género discursivo del texto, es decir, reconocer las estructuras textuales de los mismos con el propósito de anticipar el tipo de información, acontecimiento o hecho que entregará, además de su organización. Un segundo tipo se relaciona con la necesidad de determinar la meta de la lectura, permitiendo con ello focalizar los esfuerzos para dirigir la atención a aquello que se pretende lograr, es decir, cuál es el propósito de la lectura. Una tercera estrategia implicaría la activación de conocimientos previos que permitiría acceder a las ideas o información que el aprendiz sabe respecto del texto o de los contenidos de este, albergados en sus esquemas o representaciones mentales y que serían clave para ayudarle a entender y otorgar significado a aquello que lee. Finalmente, se identifica la importancia de establecer predicciones de las ideas del texto mediante la generación de preguntas y/o hipótesis que permitan a los lectores una mayor implicación cognitiva y motivacional, mejorando el recuerdo y los procesos atencionales.

Estas estrategias deberían enseñarse considerando ciertos aspectos que forman parte de los contextos de aula. En este sentido, se asume que los procesos de enseñanza-aprendizaje están regulados por 
normas y reglas básicas de comunicación y, por tanto, se sustentan en una naturaleza colaborativa que permite negociar y compartir significados. Además, dichos procesos son dinámicos, construidos a partir de interacciones entre el profesor y los estudiantes, y cuyo resultado debiera ser un incremento de la participación del estudiante con una apropiación creciente del contenido o material de la lectura (Candela, 2001). Si consideramos esto, la planificación y ejecución de los procesos de enseñanza en las aulas debería incluir acciones intencionadas que permitan apoyar y andamiar el aprendizaje, es decir, propiciar la creación de contextos cognitivos preparando al estudiante para evocar sus conocimientos previos y dotar de un sentido a la tarea de leer y comprender, activando procesos motivacionales y volitivos e implicándose afectiva y cognitivamente en dicha tarea (Borzone, 2005; Edwards y Mercer, 1988; Sánchez et al., 2010).

Al respecto, Graves y Graves (2003; Clark \& Graves, 2005) plantean la necesidad de que el diseño de experiencias andamiadas de lectura incluya dos dimensiones principales: la planificación y la implementación de dichas experiencias. En relación con la planificación, estos autores sostienen la necesidad de incorporar técnicas, estrategias y actividades en cada una de las fases de la lectura, para apoyar las necesidades de los estudiantes. En esta misma línea, autores como Solé (2008) han propuesto la incorporación de preguntas o estrategias didácticas que beneficien la comprensión alcanzada por los estudiantes, dado que la forma de organizar la secuencia de actividades en las aulas definiría, en parte, los apoyos cognitivos y los modos de participación de profesores y estudiantes (Iturra, 2013; Stodolsky, 1991).

Por otro lado y considerando la implementación de estas experiencias en los contextos de aula, Graves y Graves (2003) señalan que sería necesaria la inclusión de tareas específicas que permitan apoyar el proceso comprensivo de los estudiantes. Además de ello, la investigación en procesos de enseñanza-aprendizaje de la comprensión de textos plantea que el diálogo sería el vehículo que permitiría la construcción de significados, por parte del aprendiz (Murphy, Wilkinson, Soter, Hennessey \& Alexander, 2009), dado que este se constituiría en un instrumento de mediación (Vygostky, 1978). En este sentido, Gavelek y Raphael (1985) plantearon que el cuestionamiento es un importante recurso metacognitivo mediante el cual se fomenta la compresión y la capacidad representacional mientras se lee. 
En consecuencia, cuando se plantea la necesidad de enseñar y modelar estrategias metacognitivas en las aulas, se debe considerar la inclusión de una gama de acciones relacionadas tanto con la forma de estructurar las actividades y tareas en el momento antes de la lectura, como con propiciar interacciones comunicativas que permitan a los estudiantes participar activamente de la construcción y apropiación de tales estrategias.

\section{Metodología}

\subsection{Objetivos}

La presente investigación de carácter descriptivo tuvo como propósito caracterizar la interacción profesor-alumnos de educación media en clases dedicadas a la enseñanza-aprendizaje de la comprensión de textos e identificar la forma en que los profesores intencionan la enseñanza de estrategias metacognitivas. Para ello, el análisis se focalizó en describir la secuenciación de las actividades, interacciones discursivas y las representaciones explícitas elaboradas por los participantes de las clases, en el momento antes de la lectura.

\subsection{Participantes}

La muestra estuvo constituida por 30 grabaciones de clases de enseñanza media, 15 del año 2009 y 15 del año 2013, las cuales formaban parte del proceso de evaluación del desempeño docente chileno. Las grabaciones corresponden al módulo 2 del instrumento de evaluación denominado Portafolio (Ministerio de Educación de Chile, Mineduc, 2004) y consiste en una sesión de clase grabada con una duración de 40 minutos, en la que participa el profesor y sus estudiantes. La distribución de los niveles de enseñanza se describe en la Tabla 1.

Tabla 1. Caracterización de las sesiones, de acuerdo con el grado o nivel de enseñanza.

\begin{tabular}{|l|c|c|c|c|}
\hline & \multicolumn{4}{|c|}{ Niveles enseñanza } \\
\hline & $1^{0}$ medio & $2^{\circ}$ medio & $3^{\circ}$ medio & $4^{\circ}$ medio \\
\hline Muestra 2009 & 4 & 6 & 4 & 1 \\
\hline Muestra 2013 & 2 & 3 & 5 & 5 \\
\hline Total & 6 & 9 & 9 & 6 \\
\hline
\end{tabular}

Fuente: Elaboración propia. 
Para la selección de las sesiones, se estableció como criterio muestral intencional (Flick, 2012) que el proceso de enseñanza estuviera intencionado hacia el aprendizaje de la comprensión de textos y que correspondiera a algún nivel de enseñanza media, es decir, los últimos cursos de educación secundaria obligatoria $\left(1^{\circ}, 2^{\circ}, 3^{\circ}\right.$ y $4^{\circ}$ año medio) donde la edad promedio de los estudiantes oscila entre los 14 y los 19 años.

\subsection{Sistema de análisis}

Para describir y caracterizar las actividades, diálogos y representaciones explícitas desarrolladas antes de la lectura, se utilizó el sistema de análisis de la interacción profesor-alumno en clases de comprensión de textos propuesto por Sánchez et al. (2010), quienes han diseñado un modelo de análisis que permite desfragmentar la interacción discursiva en las aulas, operando con diferentes unidades de análisis que buscarían identificar el tipo de acción que se desarrolla en dichos espacios y las negociones que construyen los participantes, mediante sus intercambios comunicativos orientadas a la co-construcción del conocimiento. De esta forma, se pretende describir qué tipo de actividad es la más característica del proceso interaccional, el patrón de negociación en dichas actividades y el tipo de contenido negociado. Dicho sistema cuenta con un procedimiento validado (Sánchez, García, Rosales, De Sixte, y Castellano, 2008).

Particularmente para este estudio, se utilizaron las unidades de análisis de episodios y ciclos. Los primeros, se pueden definir como segmentos de actividad e intercambios comunicativos que tienen un propósito común y reconocido por los participantes, que propicia ciertas estructuras de participación. Dentro de los episodios que se han identificado en distintas investigaciones (Bustos, Montenegro, Tapia y Calfual, 2017; Ruano, Ciga, Sánchez y García, 2011), los más usuales serían: planificación, conocimientos previos y lectura e interpretación, entre otros. Así, la meta de un episodio de planificación implicaría establecer el propósito de la lectura, así como los recursos y esfuerzos necesarios para lograrla, mientras que un episodio de conocimientos previos se orientaría a activar las representaciones que poseen los estudiantes frente al tema o al tipo de texto.

La segunda unidad de análisis, los ciclos, se definen como intercambios comunicativos entre los participantes de la interacción, así como el discurso del profesor, dirigidos a elaborar o exponer una idea o contenido 
públicamente. Dentro de ellos se destacan los monólogos, habitualmente discursos elaborados en exclusiva por los profesores, así como diálogos entre profesor y estudiantes. Estos diálogos pueden adoptar dos modos distintos: ciclos triádicos (IRE) que apuntan a evaluar las respuestas de los estudiantes que se inician con una pregunta del profesor, respuesta del estudiante y la valoración de esa respuesta por parte del profesor; o los ciclos IRF (Wells, 1993) que consisten en el inicio del profesor, la respuesta del alumno y la retroalimentación del profesor que, generalmente, permite una continuidad en el proceso de negociación.

Finalmente, para caracterizar las representaciones elaboradas por los participantes en los episodios de planificación y conocimientos previos, se utilizaron las siguientes categorías de análisis.

Tabla 2. Categorías de análisis episodios.

\begin{tabular}{|c|c|c|c|}
\hline \multicolumn{4}{|c|}{ Contenidos } \\
\hline Tipo episodio & Categoría & Definición & Ejemplo \\
\hline \multirow[t]{3}{*}{ Planificación } & Objetivo & $\begin{array}{l}\text { Explicitación de } \\
\text { los objetivos de la } \\
\text { sesión. }\end{array}$ & $\begin{array}{l}\text { Profesora: Vamos a interpretar } \\
\text { un texto dramático conside- } \\
\text { rando conflictos, personajes y } \\
\text { contextos de producción. }\end{array}$ \\
\hline & Tema & $\begin{array}{l}\text { Explicitación del } \\
\text { tema de la clase o } \\
\text { lectura. }\end{array}$ & $\begin{array}{l}\text { Profesora: Vamos a reflexionar } \\
\text { sobre el viaje como metáfora } \\
\text { humana }\end{array}$ \\
\hline & Estrategia & $\begin{array}{l}\text { Explicitación de } \\
\text { las estrategias y/o } \\
\text { medios a utilizar } \\
\text { durante la lectura. }\end{array}$ & $\begin{array}{l}\text { Profesor: Vamos a realizar } \\
\text { una actividad donde tendrán } \\
\text { que identificar el tipo de texto, } \\
\text { después se señalará una parte } \\
\text { en que tendrán que interpretar } \\
\text { y aplicar los conceptos que } \\
\text { hemos trabajado. }\end{array}$ \\
\hline \multirow[t]{3}{*}{$\begin{array}{l}\text { Conocimientos } \\
\text { previos }\end{array}$} & Tipo texto & $\begin{array}{l}\text { Alusión al tipo de } \\
\text { texto que se leerá } \\
\text { en la sesión. }\end{array}$ & $\begin{array}{l}\text { Profesora: ¿Qué es una } \\
\text { narración? } \\
\text { Alumno: Cuando el autor crea } \\
\text { una historia, un cuento que } \\
\text { puede ser narrado en tercera } \\
\text { persona, segunda o primera. }\end{array}$ \\
\hline & $\begin{array}{l}\text { Tema } \\
\text { texto }\end{array}$ & $\begin{array}{l}\text { Alusión al tema del } \\
\text { texto a leer. }\end{array}$ & $\begin{array}{l}\text { Profesora: Esta imagen que } \\
\text { se refleja en la diapositiva, ¿la } \\
\text { relacionan con mito o leyenda? }\end{array}$ \\
\hline & Estrategia & $\begin{array}{l}\text { Alusión a estrate- } \\
\text { gias o medios que } \\
\text { pueden utilizarse } \\
\text { en el transcurso de } \\
\text { la lectura. }\end{array}$ & $\begin{array}{l}\text { Vamos a formular hipótesis } \\
\text { de la lectura, que ya estamos } \\
\text { acostumbrados a hacerlo. }\end{array}$ \\
\hline
\end{tabular}

Fuente: Elaboración propia. 


\subsection{Procedimiento y análisis de datos}

Seleccionada la muestra, se transcribieron literalmente las sesiones, respetando los distintos turnos de habla, es decir, las intervenciones de profesores y estudiantes. Una vez transcritas las 30 sesiones, se procedió a segmentar los intercambios comunicativos. Para ello, cada sesión se dividió en las tres fases o momentos de lectura: antes, durante y después (Clark \& Graves, 2005; Solé, 2008). Se definió que el momento antes de la lectura incluiría todas las tareas y actividades anteriores a la acción concreta de leer un texto. Una vez identificado dicho momento se operó con los episodios, agrupando las distintas tareas e intercambios comunicativos de acuerdo con la meta característica de cada uno.

Identificados los episodios, se confeccionaron los mapas de episodios, que son representaciones gráficas que permiten observar la organización temporal y secuencial de las acciones. Posteriormente, se analizaron los episodios de planificación y conocimientos previos, de acuerdo con los distintos tipos de ciclos y el tipo de elaboración pública que fueron objeto de atención o negociación entre los participantes.

Una vez categorizados los ciclos y contenidos, se sumaron las frecuencias para cada uno de los grupos y, a partir de ellas, se calcularon porcentajes. Para identificar la existencia de diferencias significativas entre los ciclos y las categorías de análisis en los episodios de planificación y conocimientos previos, entre el grupo de sesiones pertenecientes al año 2009 y el grupo perteneciente al año 2013, se utilizó el estadístico Chi cuadrado con un nivel de significancia del $0,01(\alpha=0,01)$. En caso de que algunas de las frecuencias fueran menores a 5 o igual a 0 , se adecuó el resultado mediante la corrección de Yates.

Finalmente, los criterios de confiabilidad (Guba, 1981) utilizados en la investigación fueron: la credibilidad — que se logró mediante el registro en audio de las sesiones-y la transcripción literal de las mismas, permitiendo el acceso constante a la fuente primaria de datos. La transferabilidad se alcanzó a partir de los criterios muestrales intencionales. Por último, la dependencia fue incluida mediante dos mecanismos: el primero consistió en la utilización de un libro de códigos que permitió identificar los distintos criterios para llevar a cabo el análisis; y el segundo se efectuó a partir de la triangulación de criterios de las investigadoras mediante la revisión de los procesos de segmentación y categorización. 


\section{Resultados}

\subsection{Organización temporal y secuencial}

Se identificó un total de 94 episodios en ambos grupos de clases, 47 de ellos en las sesiones del año 2009 y 47 en las sesiones del año 2013. Si bien se detectaron variaciones en la secuenciación de estos episodios, así como en la temporalidad de los mismos en cada una de las sesiones, en todos ellos se incluyeron tareas de planificación y activación de conocimientos previos, tal y como se aprecia en las Figuras 1 y 2.

Figura 1.

Mapa de episodios sesiones año 2009.

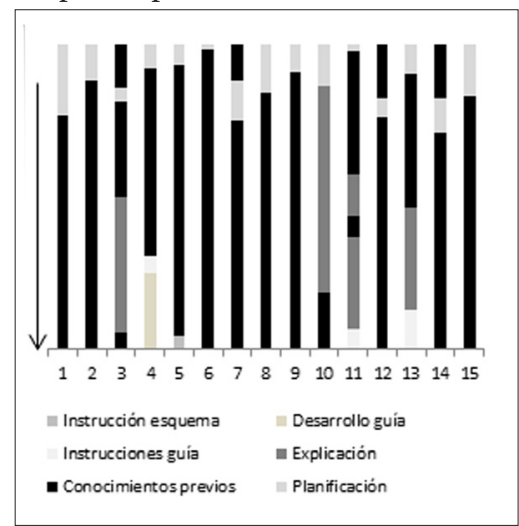

Fuente: Elaboración propia.
Figura 2.

Mapa de episodios sesiones año 2013.

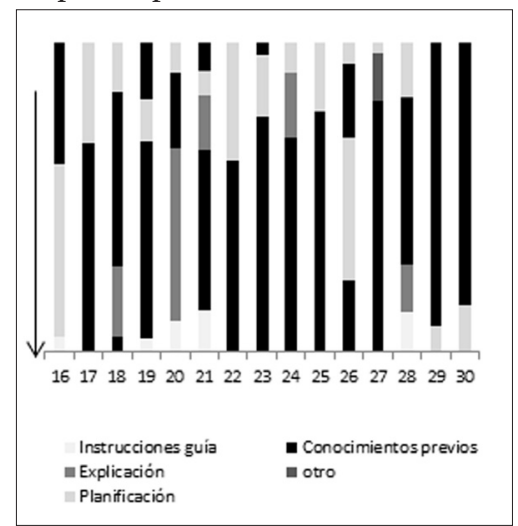

Fuente: Elaboración propia.

Las figuras anteriores indicarían que en todas las sesiones se desplegaron acciones para construir el contexto necesario para abordar posteriormente la tarea de lectura, no obstante, se distinguieron ciertas diferencias tanto en la temporalidad y secuencialidad de las acciones, como en la complejidad de las mismas. De esta manera, fue posible identificar patrones simples y complejos.

Los primeros se constituían a partir de dos episodios base, planificación y conocimientos previos reconociéndose, además, ciertas diferencias en el modo de organizar secuencialmente las actividades y tareas. Así, dentro de este patrón se pudo apreciar:

- Patrón simple lineal: articulado en base a un episodio de planificación seguido de un episodio de conocimientos previos, denotando esto que "establecemos la meta y luego activamos las representaciones que poseemos de texto". Un ejemplo de estas son las sesiones 1017. 
- Patrón simple invertido: la secuencia de acciones comenzaba con la activación de conocimientos previos y posteriormente la planificación, es decir, "primero recordamos qué sabemos del texto y después explicitamos la meta”. Las sesiones 29 y 30 son una muestra de ello.

- Patrón simple intercalado: la organización en este patrón implicaba la inclusión de dos episodios de conocimientos previos y/o episodios de planificación intercalados con otro del patrón simple. Por ejemplo, en la sesión 12 la secuencia inició con una activación de conocimientos previos, posteriormente una planificación de la sesión, para finalizar con otra activación de conocimientos previos, implicando ello que "activamos lo que sabemos, establecemos la meta y después volvemos a revisar lo que sabemos del texto". Otros ejemplos de sesiones son la 14 o la 23 .

Por otro lado, los patrones complejos incluyeron, además de los episodios base, otras acciones dedicadas a la explicación de contenidos nuevos, o bien, a entregar instrucciones acerca de la elaboración de una tarea o mapa conceptual. En ese patrón se observaron dos variantes:

- Patrón complejo de tres episodios: la secuencia de acciones incluía la activación de conocimientos previos y planificación, además de otro episodio vinculado con la explicación de un nuevo contenido o la entrega de instrucciones referidos a una tarea o mapa conceptual que deberían elaborar posteriormente, implicando ello que "establecemos una meta, revisamos lo que sabemos del texto que leeremos e incluimos una explicación acerca de un nuevo contenido". Las sesiones 3 y 18 son ejemplos de esto.

- Patrón complejo de cuatro episodios o más: este patrón se caracterizó por la inclusión de variadas actividades relacionadas con la inclusión de los episodios base más las acciones destinadas a explicar un nuevo contenido y a entregar instrucciones respecto de la tarea que realizarían posteriormente. Ejemplos de estas son las sesiones 13, 21 y 28.

Por tanto, cada uno de estos patrones representó la forma en que se desarrolló el momento antes de la lectura. Al establecer una diferenciación entre los patrones podemos observar una distribución similar entre los patrones simples y complejos presentes en cada uno de los grupos y entre las muestras del año 2009 y 2013, tal y como se resume en la Tabla 3. 
Tabla 3. Patrones identificados en momento antes de la lectura.

\begin{tabular}{|c|c|c|c|c|c|c|c|c|c|c|}
\hline \multicolumn{11}{|c|}{ Patrones } \\
\hline & \multicolumn{6}{|c|}{ Simple } & \multicolumn{4}{|c|}{ Complejo } \\
\hline & \multicolumn{2}{|c|}{ Lineal } & \multicolumn{2}{|c|}{ Invertido } & \multicolumn{2}{|c|}{ Intercalado } & \multicolumn{2}{|c|}{$\begin{array}{l}\text { Tres } \\
\text { episodios } \\
\text { distintos }\end{array}$} & \multicolumn{2}{|c|}{$\begin{array}{l}\text { Cuatro } \\
\text { o más } \\
\text { episodios } \\
\text { distintos }\end{array}$} \\
\hline & $N$ & $\%$ & $N$ & $\%$ & $N$ & $\%$ & $N$ & $\%$ & $N$ & $\%$ \\
\hline 2009 & 6 & $40 \%$ & & & 3 & $20 \%$ & 3 & $20 \%$ & 3 & $20 \%$ \\
\hline 2013 & 3 & $20 \%$ & 2 & $13 \%$ & 2 & $13 \%$ & 4 & $27 \%$ & 4 & $27 \%$ \\
\hline Total & \multicolumn{2}{|c|}{9} & \multicolumn{2}{|c|}{2} & \multicolumn{2}{|c|}{5} & \multicolumn{2}{|c|}{7} & \multicolumn{2}{|c|}{7} \\
\hline
\end{tabular}

Fuente: Elaboración propia.

Los datos aportados en la tabla anterior indicarían que en todas las sesiones, independientemente de los años, los profesores incorporaron acciones que buscarían estructurar el proceso de lectura, incorporando acciones orientadas a planificar la lectura y a activar conocimientos previos y, en algunos casos, otras actividades relacionadas con la posterior lectura. No obstante, es necesario identificar cómo se construyeron en la interacción discursiva tales acciones, así como las elaboraciones públicas que se relevaron en el proceso interactivo, para describir si efectivamente la ejecución de estas acciones estuvo orientada a trabajar aspectos vinculados con el modelaje de la metacomprensión.

\subsection{Episodios de planificación}

Se identificó un total de 31 episodios de planificación (15 en las muestras del año 2009 y 16 en las muestras del año 2013). El número de ciclos que se desplegó a lo largo de la interacción fue similar (16 en el año 2009 y 19 en el año 2013). De igual modo, el tipo de ciclo no presentó grandes variaciones, siendo la mayoría de ellos de carácter monologal en ambas muestras. En otras palabras, la acción de planificar la lectura dependió del profesor, tal y como se señala en los Gráficos 1 y 2. 
Gráfico 1.

Porcentajes de tipos de ciclos episodios planificación.

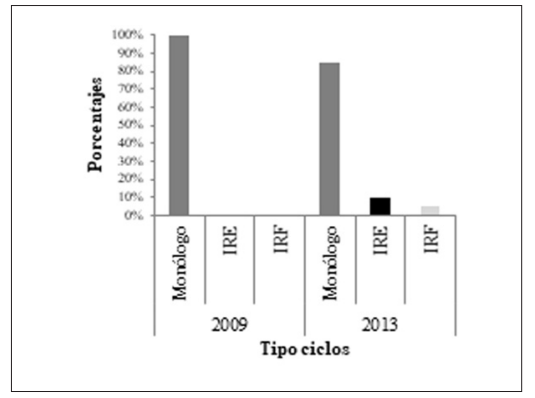

Fuente: Elaboración propia.

\section{Gráfico 2.}

Porcentaje de categorías contenido público episodios planificación.

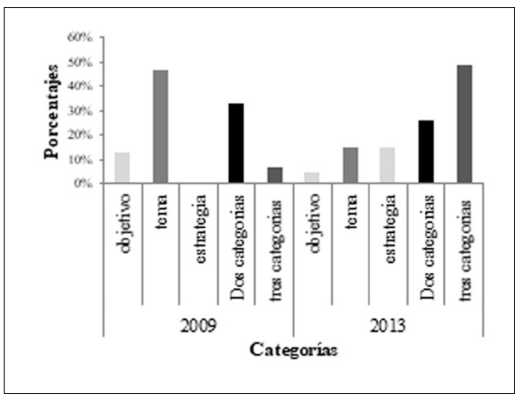

Fuente: Elaboración propia.

Por otra parte, las elaboraciones o contenidos públicos elicitados en la acción discursiva de los profesores mostraron variaciones tanto entre los grupos como entre las muestras, como se observa en el Gráfico 2.

Ahora bien, si analizamos las categorías se desprende, en primer lugar, una diversidad en las elaboraciones que se explicitaron en estos episodios, desde una alusión al tema de la sesión u objetivo como:

Profesora: el objetivo de aprendizaje de nuestra clase de hoy será identificar tipos de descripciones como una forma básica de texto expositivo

hasta la inclusión de monólogos en los que se entregaba el tema de la clase, el objetivo y los medios para alcanzarlo:

Profesor: nuestro objetivo ... reconocer el contenido de los textos que leen y que se usan para la exposición de temas de actualidad ... el tema del cual trata el texto que vamos a leer hoy se titula El mall, la cultura del consumismo ... lo que vamos a realizar durante el desarrollo de nuestra clase es leer atentamente el texto, analizarlo, interpretarlo y luego responder las preguntas que aparecen en él.

En segundo lugar, se apreció en el grupo de sesiones del año 2009 una mayor concentración en episodios que explicitaron una sola categoría, mientras que aquellos en las que se construyó la meta de la lectura considerando el tema, los objetivos y los medios fue menor. Contrariamente, en las sesiones del año 2013, esta situación se invirtió, observándose un mayor número de episodios en los que se aludió a las 
tres categorías y una disminución de sesiones que solo abordaron la construcción de la meta a partir de una sola.

En síntesis, en los episodios de planificación de las sesiones de los años 2009 y 2013 se apreció la inclusión de elaboraciones públicas vinculadas con estrategias metacognitivas, aun cuando estas fueron en su mayoría de carácter monologal. Además, se observó una heterogeneidad en la elaboración de contenidos, considerando cada una de las sesiones, y los grupos de muestras.

\subsection{Episodios de conocimientos previos}

Un total de 39 episodios de conocimientos previos fue identificado en el momento antes de la lectura. Estos episodios albergaron 249 ciclos, 137 ciclos en la muestra del año 2009 y 112 en las muestras del año 2013. La mayoría de estos ciclos fue de tipo IRF, es decir, el profesor elaboró una pregunta y tras la respuesta del estudiante, se construyó un diálogo que propició la activación de los conocimientos previos. En este sentido, gran parte del tipo de comunicación que se suscitó durante la interacción fue dialógica, en tanto los ciclos de carácter monologal representaron una proporción menor, como se parecía en los Gráficos 3 y 4. En otras palabras, las acciones durante estos episodios fueron construidas entre profesores y estudiantes y esta tendencia se mostró homogénea en las dos muestras.

\section{Gráfico 3.}

Porcentajes de tipos de ciclos episodios conocimientos previos.

\section{$\mathrm{X} 2=1,76 ; \mathrm{GL}=2 ; p=0,41478291$.}

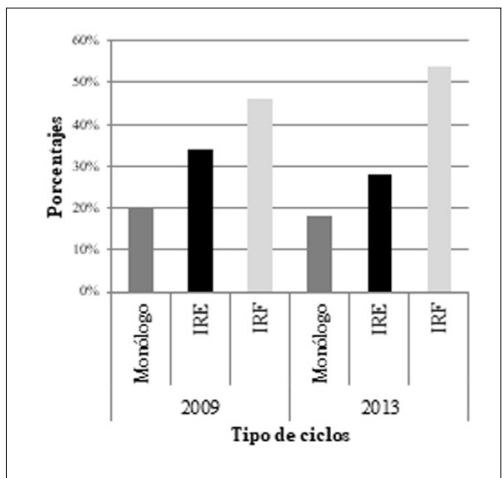

Fuente: Elaboración propia.

\section{Gráfico 4.}

Porcentaje de categorías contenido público episodios conocimientos previos.

Yates' $\mathrm{x} 2=0,566 ; \mathrm{GL}=2 ; p=0,45426024$.

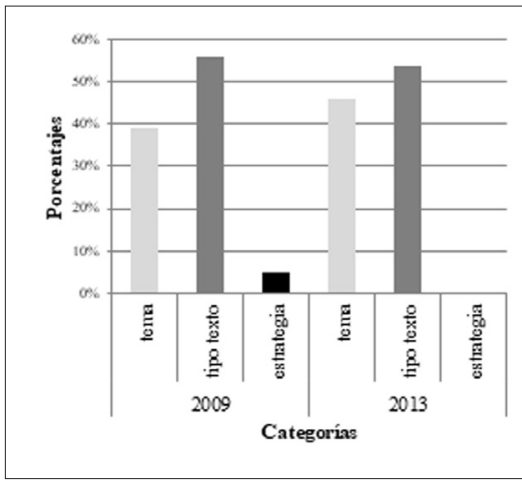

Fuente: Elaboración propia. 
Por otra parte y en relación con los contenidos elaborados públicamente se observó, tal y como se indica en el Gráfico 4, que la mayoría de ellos se concentró en las categorías tema texto, independientemente del año de la muestra:

Profesor: ¿Qué sentimientos le produce al hombre toda esta problemática de guerras, de conflictos bélicos, morales, de cambio en el mundo? ¿Cuál sería el sentimiento? ¿Qué les sucede?

Alumno: Miedo.

Profesora: Miedo.

y tipo de texto:

Profesora: ¿Qué es una columna de opinión?

Alumno: Es un texto de crítica

Profesora: Es un texto de crítica, ya.

Lo anterior indicaría que, en general, los profesores tendieron a elicitar conocimientos de los estudiantes vinculados con las características del texto y la información que ellos recordaban respecto de la temática del mismo, mientras que la activación de recuerdos relacionados con estrategias o las formas en las que han logrado enfrentar anteriormente la lectura mientras la ejecutaban, representaron un porcentaje considerablemente menor e inexistente en el caso de la muestra del año 2013. Considerando ambos elementos, se puede señalar que la mayoría de los episodios de conocimientos previos se construyó a partir de una interacción dialógica en la que se privilegiaron las ideas previas que los estudiantes tenían del texto como contenido a trabajar.

\section{Conclusiones}

La pregunta inicial que buscaba responder el presente trabajo era caracterizar la interacción profesor-alumnos identificada en clases de comprensión de textos en enseñanza media, con el propósito de describir cómo los profesores promovían con sus prácticas, la enseñanza y modelaje de las estrategias metacognitivas. Para ello, se describió la forma en que se estructuró el momento antes de la lectura, a partir de la inclusión y secuenciación de distintas actividades vinculadas con la planificación de la lectura, además de identificar el tipo de interacción discursiva y las elaboraciones públicas producto de estas interacciones. 
En primer lugar - y de acuerdo con lo propuesto por los trabajos de Graves y Graves (2003) y Solé (1998) - se puede señalar que todas las sesiones incluyeron acciones dirigidas a construir el contexto cognitivo (Borzone, 2005; Sánchez et al., 2010) necesario para planificar la lectura de los textos. En este sentido, se desarrollaron tareas de planificación y activación de conocimientos previos, las que variaron en algunos casos en la secuencialidad de dichas acciones y en la inclusión de una actividad destinada a trabajar un nuevo conocimiento relacionado con los contenidos propios del currículo chileno. Respecto de este punto en particular, se debe considerar que las sesiones se encuentran ubicadas en los cursos secundarios y, por tanto, los objetivos de aprendizaje declarados implicarían la profundización de temáticas vinculadas con las características de los tipos de textos. Por otra parte, no se identificaron variaciones entre los años, lo que podría suponer que la práctica de los profesores al planificar y ejecutar esta fase antes de la lectura, no ha tenido cambios en los últimos 5 años.

Retomando el resultado de la práctica de estos profesores, que representaría la secuencia de acciones necesarias para organizar y planificar el proceso comprensivo "establecemos un objetivo y activamos conocimientos previos", se podría suponer que sería un paso en la formación de lectores estratégicos (Paris et al., 1991), no obstante, la forma en que finalmente se desarrolló la construcción de este contexto cognitivo en las aulas implicó, en algunos casos, un porcentaje mayor de responsabilidad por parte de los profesores.

Así y considerando los episodios de planificación, los resultados mostraron que la mayor parte de los patrones comunicativos fueron monologales, es decir, gran parte de las elaboraciones explícitas relacionadas con identificar la meta o propósito de lectura, la elaboración de hipótesis respecto de la misma y la explicitación de los medios o pasos para organizar el proceso (Block \& Presley, 2007) fueron planteados casi exclusivamente por los profesores, impidiendo con ello que los estudiantes opinaran o participaran en la construcción de los mismos (Candela, 2001). Aun así, se observó una mayor riqueza en la inclusión de una variedad de estrategias metacognitivas como la elaboración de un objetivo preciso, la focalización en el tema de la lectura y los medios para llevar a cabo la tarea en parte de las sesiones del año 2013, en comparación con las del año 2009. Ello plantearía un avance en la práctica educativa de los profesores al abordar la activación de los 
procesos necesarios para la regulación de la tarea de lectura, sin embargo, estas acciones fueron mayoritariamente monologales.

Por otra parte - y en relación a la activación de conocimientos previos-, se identificó que los patrones comunicativos característicos de esta acción variaron respecto de los episodios de planificación, dado que se construyeron en su mayoría a partir del diálogo entre profesores y estudiantes, permitiendo esto una mayor participación de los estudiantes en la identificación de la información necesaria para relacionarla con las nuevas ideas de los textos (Gavelek \& Raphael, 1985). Además, gran parte de las ideas previas evocadas por los estudiantes se vinculaban directamente con el texto que leerían a continuación, ya sea a partir de conocimientos previos relacionados con el tema o el tipo de texto. Si bien, ello implicaría promover una mayor capacidad representacional (Brown, 1987) al activar aquello que sabemos respecto de los textos que se leerán (Solé, 2008), la capacidad para identificar estrategias utilizadas anteriormente como apoyo a la planificación y monitoreo de su propia comprensión (Baker \& Carter, 2009), fue escasa o nula en ambos grupos y, por tanto, la capacidad de explicitar y apropiarse de aquellas estrategias para autorregular su propio proceso no fue relevada como algo fundamental.

Considerando la práctica de estos profesores como ejemplo del trabajo que se desarrolla en las aulas cuando estudiantes y profesores leen conjuntamente (Sánchez et al., 2010), se puede plantear que los profesores incluyeron acciones para organizar estas actividades conjuntas, y que estas se orientaron, en parte, a modelar estrategias para planificar el momento antes de la lectura (Stodolsky, 1991) apoyando así el proceso comprensivo, sin embargo, se requiere la participación activa e intencionada de los aprendices para que estos logren apropiarse de las estrategias metacognitivas y, para ello, es necesaria la inclusión de espacios de mayor diálogo, reconociendo que este es un instrumento de mediación que permite a los estudiantes implicarse en su propio proceso (Murphy et al., 2009), además de incluir mayor explicitación a las estrategias que utilizan efectivamente los aprendices para apoyar su comprensión de textos.

A modo de cierre y reflexión, indudablemente el desafío de lograr alfabetizar a los estudiantes de la región (Unesco, 2015) implica mejorar los procesos de enseñanza en la comprensión de textos, permitiendo potenciar la capacidad estratégica de los lectores y, para ello, también es 
indispensable que se desarrollen líneas de apoyo y asesoramiento a los profesores, de modo que estos puedan apropiarse de métodos y estrategias actualizadas de enseñanza que permitan mejorar su propia práctica.

\section{Referencias bibliográficas}

Anderson, R. \& Pearson, D. (1984). A schema-theoretic view of basic processes in reading comprehension. En D. Pearson (Ed.), Handbook of reading research (pp. 255-291). New York: Longman.

Baker, L. \& Carter, L. (2009). Metacognitive processes and reading comprehension. En S. Israel \& G. Duffy (Eds.), Handbook of research on reading comprehension (pp. 373-388). New York, London: Routledge.

Block, C. \& Pressley, M. (2007). Best practices in teaching comprehension. En L. B. Gambrell, M. Morrow, \& M. Pressley (Eds.), Best practices in literacy instruction ( $3^{\text {rd }}$ ed.) (pp. 220-242). New York: Guilford.

Borzone, A. (2005). La lectura de cuentos en el jardín infantil: un medio para el desarrollo de estrategias cognitivas lingüísticas. Psykhe (Santiago), 14(1), 193-209. https://doi.org/10.4067/ $\underline{\text { s0718-22282005000100015 }}$

Brown, A. (1987). Metacognition, executive control, self-regulation and other more mysterious mechanisms. En F. Weinert \& R. Kluwe (Eds.), Metacognition, motivation and understanding (pp. 65116). Hillsdale, NJ: Lawrence Erlbbaaum Associates.

Bustos, A., Montenegro, C., Tapia, A., y Calfual, K. (2017). Leer para aprender: cómo interactúan los profesores con sus alumnos en la educación primaria. Ocnos. Revista de Estudios Sobre Lectura, 16(1) 89-106. https://doi.org/10.18239/ocnos 2017.16.1.1208

Candela, A. (2001). Corrientes teóricas sobre discurso en el aula. Revista Mexicana de Investigación Educativa, 6(12), 317 333. Recuperado de http://www.redalyc.org/articulo. oa?id=14001208

Clark, K. \& Graves, M. (2005). Scaffolding students' comprehension of text. The Reading Teacher, 58(6), 570-580. https://doi.org/10.1598/ $\underline{\mathrm{rt} .58 .6 .6}$ 
Edwards, D. y Mercer, M. (1988). El conocimiento compartido. El desarrollo de la comprensión en el aula. Barcelona: Paidós.

Flick, U. (2012). Introducción a la investigación cualitativa. Madrid: Editorial Morata.

Flavell, J. (1979). Metacognition and cognitive monitoring. A new area of cognitive-developmental inquiry. American Psychologist, 34(10), 906-911. https://doi.org/10.1037/0003-066x.34.10.906

Gavelek, J. \& Raphael, T. (1985). Metacognition, instruction, and the role of questioning activities. En D. L. Forrest-Pressley, G. E. MacKinnon, \& T. Gary Waller (Eds.), Metacognition, cognition, and human performance (pp. 103-135). San Diego, CA: Academic Press.

Graves, M. \& Graves, B. (2003). Scaffolding reading experiences: Designs for student success. Norwood, MA: Christopher-Gordon.

Guba, E. (1981). Criteria for assessing the trustworthiness or naturalistic inquiries. Educational Communication and Technology, 29(2), 75-91. Recuperado de http://www.jstor.org/ stable/30219811?origin=JSTOR-pdf

Iturra, C. (2013). Los diálogos construidos y los contenidos elaborados en clases dedicadas a la comprensión de textos, en aulas chilenas. Psicología educativa, 19(2), 113-122. https://doi.org/10.1016/ $\underline{\text { s1135-755x(13)70018-6 }}$

Kintsch, W. (1998). Comprehension: A paradigm for cognition. Cambridge, UK: Cambridge University Press.

Madariaga, J. M. y Martínez-Villabeita, E. (2010). La enseñanza de estrategias de comprensión y metacomprensión lectora: un programa implementado por el profesorado. Anales de Psicología, 26(1), 112-122. Recuperado de https://dialnet.unirioja.es/servlet/arti culo? codigo $=3133192$ \&orden $=236147$ \&info=link

Ministerio de Educación de Chile, Mineduc. (2004). Reglamento sobre evaluación docente. Santiago de Chile: Autor.

Murphy, P. K., Wilkinson, I. A., Soter, A. O., Hennessey, M. N., \& Alexander, J. F. (2009). Examining the effects of classroom discussion on students' comprehension of text: A meta-analysis. Journal of Educational Psychology. 101(3), 740-764. https://doi. org/10.1037/aoo15576 
Myers, J. \& O'brien, E. (1998). Accessing the discourse representation during reading. Discourse Processes, 26(2-3), 131-157. https://doi. org/10.1080/01638539809545042

Organización para la Cooperación y el Desarrollo Económicos, OCDE (2006). Marco de la evaluación. Conocimientos y habilidades en ciencias, matemáticas y lectura. Recuperado de http://www. oecd.org/dataoecd/59/2/39732471.pdf

Paris, S., Wasik, B., \& Turner, J. (1991). The development of strategic readers. En R. Barr, M. Kamil, P. Mosenthal, \& D. Pearson (Eds.), The handbook of reading research (Vol. 2, pp. 609-640). New York: Longman.

Ruano, E., Ciga, E., Sánchez, E., y García J. (2011). Un protocolo para observar cómo los profesores ayudan a sus alumnos cuando leen textos en el aula. Psicología Educativa, 17(2), 127-145. https://doi.org/10.5093/ed2011v17n2a2

Sánchez, E., García, R., Rosales, P., De Sixte, R., y Castellano, N. (2008). Elementos para analizar la interacción entre estudiantes y profesores: ¿qué ocurre cuando se consideran diferentes dimensiones y diferentes unidades de análisis? Revista de Educación, 346, 105-138. Recuperado de https://dialnet.unirioja. es/servlet/articulo?codigo=2591907\&orden=156641\&info=link

Sánchez, E., García, R., y Rosales, J. (2010). La lectura en el aula: qué se hace, qué se debe hacer y qué se puede hacer. Barcelona: Grao.

Schmitt, M. \& Baumann, J. (1990). Metacomprehension during basal reading instruction: Do teachers promote it? Reading Research and Instruction, 29(3), 1-13. https://doi. org/10.1080/19388079009558012

Slavin, R., Lake, C., Chambers, B., Cheung, A., \& Davis, S. (2009). Effective reading programs for the elementary grades: A best-evidence synthesis. Review of Educational Research, 79(4), 1391-1465. https://doi.org/10.3102/0034654309341374

Solé, I. (1998). Estrategias de lectura. 8a edición. Barcelona: Editorial Graó.

Solé, I. (2008). Estrategias de lectura Barcelona: Graó.

Solé, I. (2012). Competencia lectora y aprendizaje. Monográfico. Revista Iberoamericana de Educación, 52, 43-61. https://rieoei.org/ historico/documentos/rie59a02.pdf 
Stodolsky, S. (1991). La importancia del contenido en la enseñanza. Actividades en las clases de matemáticas y ciencias sociales. Barcelona: Paidós.

Trabasso, T. \& Bouchard, E. (2002). Teaching readers how to comprehend text strategically. En C. Block \& M. Pressley (Eds.), Comprehension instruction: Research-based best practices (pp. 176-200). New York: Guilford Press.

United Nations Educational, Scientific and Cultural Organization, Unesco. (2015). Education for all 200o-2015: Achievements and challenges. Education for All Global. Monitoring Report 2015. Paris: Autor. Recuperado de https://unesdoc.unesco. org/ark:/48223/pf0000232205?posInSet=3\&queryId=0982e5 de-1a08-49c3-bf68-92d5e7351c40

Vygotsky, L. S. (1978). Mind in society. The development of higher psychological processes. Cambridge, MA: Harvard University Press.

Wells, G. (1993). Reevaluating the IRF sequence: A proposal for the articulation of theories of activity and discourse for the analysis of teaching and learning in the classroom. Linguistics and Education, 5(1), 1-38. https://doi.org/10.1016/ s0898-5898(05)80001-4 